\title{
Lentes violetas: la irrupción de la figura de la editora de género en medios generalistas. Estudio preliminar cualitativo en España y Estados Unidos
}

Betaurreko moreak: genero editorearen figura hedabide jeneralistetan. Espainian eta Estatu Batuetan eginiko aurretiazko azterketa kualitatiboa

Purple Lenses: The Appearance of the Gender Editor in the Mainstream Press. Preliminary Qualitative Study in Spain and the United States

\author{
Susana Pérez Soler^, Meritxell Roca Sales \\ Ramon Llull/Columbia University
}

RESUMEN: Este artículo explora el rol de la editora de género que algunos medios de comunicación han introducido recientemente en las redacciones periodísticas con un doble objetivo: incorporar la perspectiva de género en la cobertura mediática y reivindicar condiciones laborales igualitarias. El objeto de estudio son seis medios de comunicación, tradicionales y nativos digitales, de España y Estados Unidos. Se realizan entrevistas en profundidad semi-estructuradas a cinco editoras de género de estos medios con el fin de entender las funciones atribuidas a este nuevo rol y de analizar las causas de su aparición, tanto desde una perspectiva social como económica.

PALABRAS CLAVE: Editora de género (término no en thesaurus); nuevos roles profesionales (término no en thesaurus); género; periódico; feminismo.

ABSTRACT: This paper explores the newly created role of the gender editor that some media have started introducing in newsrooms with a double goal: to include the gender perspective in news coverage and to claim equal working conditions. This paper analyzes six newspapers, legacy and digital natives, from Spain and the United States. Semi-structured in-depth interviews were conducted with five gender editors to understand the responsibilities attributed to this new role as well as and the causes of their creation, both from a social and an economic perspective.

KEYWORDS: Gender editor; new professional roles; gender; newspaper; feminism.

\footnotetext{
* Correspondencia a / Corresponding author: Susana Pérez Soler. Fac. de Comunicació i Relacions Internacionals Blanquerna, Universitat Ramon Llull, Plaça Joan Corominas (08001 Barcelona) - susanaps@blanquerna.url.edu - https://orcid.org/0000-0002-2578-2761

Cómo citar / How to cite: Pérez Soler, Susana; Roca Sales, Meritxell (2019). "Lentes violetas: la irrupción de la figura de la editora de género en medios generalistas. Estudio preliminar cualitativo en España y Estados Unidos", Zer, 25(47), 65-83. (https://doi.org/10.1387/zer.20738).
}

Recibido: 5 abril, 2019; aceptado: 6 septiembre, 2019.

ISSN 1137-1102 - eISSN 1989-631X / (c) 2019 UPV/EHU

(C) (i) Esta obra está bajo una licencia 


\section{Introducción}

A finales de los años 90, coincidiendo con la consolidación de la $W W W$, los medios de comunicación, y en especial los periódicos, vivieron un momento de crisis organizativa con el auge de sus propias versiones online. Mientras que las grandes corporaciones se reinventaron a sí mismas, los nuevos medios nativos digitales se desenvolvieron con más soltura ya que estructuras y roles profesionales se modelaron ad hoc. (Anderson, Bell \& Shirky, 2012).

Numerosas investigaciones han estudiado a fondo el impacto que la digitalización tuvo (y sigue teniendo) en los medios, tanto a nivel organizativo como de gestión (Boczkowski, 2004; Deuze, 2011; Anderson, Bell \& Shirky, 2012; Pavlik, 2013; Pérez \& Micó, 2015), así como en la audiencia (Gillmor, 2004; Castells, 2009). Los medios tradicionales se tuvieron que adaptar a un nuevo contexto en el que los antiguos modelos de negocio basados en la programación única, la publicidad y la subscripción quedaron obsoletos (Roca, 2014). Internet forzó a los medios a adaptarse a una realidad incierta, cambiante y ante todo desconocida, un proceso que se ha agravado estos últimos cinco años con la consolidación de las redes sociales, gracias a la popularidad de smartphones y tabletas (Rashidian, Brown \& Hansen, 2018).

De todas las transformaciones acontecidas en la industria periodística a consecuencia de la disrupción digital, la redefinición de las relaciones entre las empresas informativas y las audiencias es quizás la más significativa, y una de las que ha recibido mayor atención por parte de los investigadores (Domingo, 2011; Holton, Lewis \& Coddington, 2016). En primer lugar, las audiencias han dejado de ser pasivas para ser activas, para interactuar con el medio. Si bien ya lo eran antes del nacimiento de los nuevos medios (a través de cartas al director o llamadas a emisoras y televisiones), ahora, con más canales para la participación, más facilidades para hacer llegar el mensaje y menos control por parte de los editores, lo son aún más. En segundo lugar, las audiencias ya no son solo consumidoras, sino también productoras. Mandan piezas escritas, fotografias o vídeos al medio digital, material que tras ser editado puede llegar a ser publicado. Y finalmente, las audiencias han dejado de ser individuos aislados para convertirse en comunidades conectadas socialmente. Los foros y chats, pero, muy especialmente, las redes sociales han posibilitado esta transformación.

Los medios se han esforzado en aproximar sus productos informativos a las audiencias incorporando nuevos roles profesionales con habilidades para compartir y promocionar contenido en las redes sociales e interactuar con los usuarios en los diferentes canales digitales del medio (Larsson \& Ihlebæk, 2017). El editor de medios sociales (social media strategist en inglés) o el administrador de comunidades (community manager en inglés), así como el técnico en SEO son 
perfiles dedicados a ello de reciente creación (Caminero-Fernández \& SánchezGarcía, 2018).

En este contexto, y al calor del caso Harvey Weinstein ocurrido en Estados Unidos en 2016 y de la huelga feminista convocada en España el 8 de marzo de 2018, las empresas periodísticas han empezado a incorporar un nuevo rol en las redacciones: la editora de género. Una figura que persigue alcanzar un mayor número de mujeres, una audiencia minoritaria para los medios generalistas (Toff \& Palmer, 2018; Zeisler, 2018).

\section{Nuevos roles profesionales}

Las tareas que realizan los periodistas, las habilidades que necesitan y el puesto que ocupan dentro de los medios han cambiado drásticamente con la consolidación de las redes sociales como canales de consumo de noticias (Bell, et. al., 2017; Digital News Report, 2017). Los medios han incorporado perfiles para distribuir los contenidos mediante las plataformas sociales con el objetivo de alcanzar a una mayor audiencia e interaccionar con ella. Se han generado puestos también para recopilar la información de los ciudadanos en las redes sociales, y para editar y modificar las contribuciones de los usuarios. Este nuevo perfil se conoce como «administrador de la comunidad» (community manager en inglés) (Cobos, 2011; Bakker, 2014).

Han aparecido nuevos roles más técnicos, como los profesionales SEO destinados a la optimización de los contenidos en los motores de búsqueda, pero también perfiles encargados de filtrar el contenido más que de producir nuevo. En un paradigma digital de exceso de información, muchos profesionales son necesarios para recolectar, gestionar y curar información más que para crear nueva (content curator en inglés) (Piet, 2012; Guallar \& Codina, 2018). También hay perfiles más analíticos que analizan cuantitativamente los datos de la audiencia para alcanzar mayores comunidades y generar más ingresos. Así, los medios han incorporado perfiles para distribuir mejor el contenido en los canales digitales y para gestionar las relaciones con dicha audiencia (Bakker, 2014).

\subsection{IRRUPCiÓN DEL ROL DE LA EDITORA DE GÉNERO}

La analítica web ha permitido segmentar con detalle la audiencia, y las empresas buscan nichos concretos a partir de la producción específica de contenidos. Recientemente, algunos medios tradicionales, han reparado en un problema: el usuario tipo que visita su página web es mayoritariamente un hombre de mediana edad. Así lo manifestaba Renée Kaplan, la primera "directora de participación de la audiencia» del Financial Times, desde junio de 2015, en una entrevista publicada por Nieman 
$\mathrm{Lab}^{1}$ "descubrimos que nuestros suscriptores eran 80 por ciento hombres y 20 por ciento mujeres ... eso no es bueno... no era un reflejo de lo que queríamos, y en un mundo en el que el 50 por ciento o más de la población son mujeres, también fue una oportunidad comercial.» No solo eso: las mujeres encontraron el tono del medio desagradable y se desconectaron de su contenido. Y cuando se les pedía, en grupos focales, que describiesen quién sería el periódico si fuera una persona, decían que sería un hombre.

Incluso en los países desarrollados, donde la equidad entre hombres y mujeres ha mejorado en los últimos años, las mujeres siguen siendo más propensas que los hombres a decir que evitan las noticias, una brecha de género que tiene implicaciones importantes para la participación política. En una investigación reciente llevada a cabo en el Reino Unido descubrieron que el rechazo de algunas mujeres a consumir noticias se debe (1) a que a menudo se perciben a través de una lente de género, es decir, en palabras de los autores del estudio: "las noticias son vistas para hombres», y (2) a que el consumo diario de los medios está sujeto a desigualdades estructurales, como, por ejemplo, las divisiones del trabajo o las cargas físicas y emocionales de las responsabilidades de cuidado, que recaen principalmente en las mujeres y pueden interferir en mantenerse al día con las noticias (Toff \& Palmer, 2018.)

Los medios ahora buscan captar la atención de las mujeres. En el caso del Financial Times conseguir una mayor audiencia femenina, según su desarrolladora de audiencias Renee Kaplan, pasa por: «un nuevo boletín dirigido a (pero no explícitamente "para») mujeres que ahora está superando a los otros boletines del FT en términos de tasa abierta. Promoción regular en toda la publicación de artículos que están sobre-indexando con mujeres. Una nueva sección de cobertura enfocada a industrias de servicios profesionales como derecho y consultoría. Y una renovación de la sección de opinión, con un llamado reescrito diseñado para alentar a las mujeres a presentar editoriales» (Hazard, 2018).

La editora de género, que ha emergido recientemente, después del caso Harvey Weinstein ocurrido en Estados Unidos en 2016 y de la huelga feminista convocada en España el 8 de marzo de 2018, tiene cierto paralelismo con el rol del editor de medios sociales, en tanto que es una figura vinculada a adecuar el producto informativo a un tipo de audiencia nicho que el medio quiere conquistar. Probablemente esta figura no existiría sin la analítica web; las nuevas tecnologías ofrecen la capacidad de conocer mejor a sus públicos y, como resultado, aparecen roles puente entre nichos de audiencia y plataformas.

\footnotetext{
$1<$ http://www.niemanlab.org/2018/04/if-the-financial-times-were-a-person-it-would-be-aman-heres-how-the-paper-is-trying-to-change-that/> [Consulta: 3 de abril de 2019]
} 


\section{Iniciativas feministas en los medios y nativos digitales feministas}

La brecha de género en los medios está bien establecida: las mujeres están subrepresentadas en las redacciones periodísticas y los roles de liderazgo son fundamentalmente de hombres (Franks, 2013; McCracken, et. al., 2018; García Saiz, 2018). Todavía existen pocos estudios sobre cómo la incorporación de las mujeres a casi todas las profesiones las ha transformado, ni en qué grado si es que lo ha hecho. En el caso de la profesión periodística, no hay evidencias de si la paulatina incorporación de las mujeres ha significado un cambio aunque estudios recientes muestran que las mujeres periodistas, al incorporarse a la profesión han adoptado uno de estos tres roles: ser una de ellos (asimilando rutinas y procedimientos profesionales sin cuestionarlos), mantener una lucha constante por incorporar una visión propia o alienarse voluntariamente y dedicarse a su manera personal de entender la profesión, sin aspiraciones de poder o cargos de responsabilidad dentro de la organización (Gallego, 2013).

Existe otra brecha igualmente bien establecida pero menos estudiada: las mujeres tienden a leer menos noticias hard (política, economía e internacional) que los hombres (Rosentiel, 2008; Benesch, 2012). Esta brecha tiene implicaciones en el negocio y es lo que busca resolver la creación del rol de la editora de género.

Los medios tradicionales, tanto en España como en Estados Unidos, incorporan estrategias editoriales para mitigar la brecha de género en el consumo de noticias. La industria de los medios está en crisis, con pérdidas financieras y de empleos, y las empresas requieren nuevas audiencias e ingresos para sobrevivir. Las mujeres representan el 50\% de la población mundial, de manera que el aumento del número de audiencia femenina podría ofrecer ingresos adicionales a partir del incremento de suscripciones e impresiones publicitarias.

The New York Times incorporó la primera editora de género, Jessica Bennett, el 30 de octubre de 2017, el día en que el diario publicó un artículo documentando las denuncias de agresión sexual contra Harvey Weinstein. Desde entonces, Bennett ha creado un boletín electrónico (El momento \#MeToo) para documentar y analizar casos sobre conducta sexual inapropiada en diferentes sectores; ha escrito sobre cómo el escándalo Weinstein ha desencadenado un movimiento global y ha organizado una serie de eventos en directo sobre el abuso masculino del poder (The New York Times, 2017). Liz Spayd, ex editora pública del mismo periódico, ya propuso en una columna de 2017 que la «escasez general de mujeres periodistas» en The New York Times podría tener un impacto «no solo en los contenidos periodísticos sino también en la audiencia del Times» (Spaid, 2017). 
En España, medios como El País, eldiario.es y RTVE también han creado esta figura. El primero en anunciarlo fue El País, que el 12 de mayo de 2018 nombraba en este cargo a la periodista Pilar Álvarez. En septiembre del mismo año lo hacía eldiario.es con Ana Requena, fundadora del reconocido blog Micromachismos, como redactora jefe de género y constituía un equipo de tres personas — contando a la misma Requena - dedicado a tratar los temas de igualdad, convirtiéndose así en el único medio generalista que cuenta con esta especificidad. En octubre, las periodistas Alicia G. Montano y Paloma Zamorano eran nombradas como primeras editoras de Igualdad de la radio y televisión públicas españolas.

Más allá de las iniciativas feministas dentro de los medios generalistas, también han aparecido, principalmente en los Estados Unidos, nativos digitales cuyo target específico son las mujeres, y más concretamente las mujeres jóvenes. Aunque los medios digitales creados específicamente para recoger las voces de las mujeres existen desde hace tiempo, ha habido un aumento de este tipo de publicaciones recientemente. The Skimm (www.theskimm.com) se lanzó en 2012 como un boletín diario para mujeres para cubrir las noticias del día. En 2015, Vice creó Broadly (https:// broadly.vice.com), cuyo objetivo es contar las experiencias de las mujeres. Lenny Letter (www.lennyletter.com), un boletín informativo sobre «feminismo, estilo, salud, política, amistad y todo lo demás» se fundó ese mismo año. En 2016, Mic lanzó The Slay (https://mic.com/slay) para cubrir los «problemas que impactan la vida de las mujeres jóvenes». Un año más tarde, en 2017, The Washington Post creó el medio The Lily, una publicación para mujeres» (Zeisler, 2017).

\section{Objetivos e hipótesis}

El objetivo principal de este estudio de caso es analizar la percepción que distintas profesionales del ámbito periodístico español y estadounidense (medios generalistas) tienen acerca de la figura de la editora de género. Nuestra hipótesis de partida apunta que la creación de este nuevo rol profesional responde a una iniciativa comercial o de marketing motivada, entre otros, por el impacto del movimiento \#metoo. Al mismo tiempo, sostenemos que la creación de contenidos editados bajo una lente de género podría conllevar cierta marginalización en términos de cobertura (jerarquía, extensión y visibilidad) así como una segmentación en términos de audiencia (lectoras fundamentalmente femeninas).

Consideramos que esta investigación, pese a sus limitaciones, hace dos aportaciones críticas para el estudio de este rol de reciente creación. Por un lado, una aproximación descriptiva y analítica de sus funciones y por otro lado una reflexión crítica acerca del oportunismo (o no) de su creación, consolidación y expansión. 


\section{Metodología y limitaciones de la investigación}

El enfoque de este artículo es cualitativo basado en entrevistas en profundidad semi-estructuradas a periodistas con funciones directamente relacionadas con la perspectiva de género (tres en España y dos en Estados Unidos). Las entrevistadas y sus cargos son: Pilar Álvarez (corresponsal de género, El País), Alicia Gómez (editora de igualdad, RTVE), Monica Hesse (gender columnist, The Washington Post), Ana Requena (redactora jefa de género, eldiario.es) y Alanna Vagianos (global women's rights and gender reporter, The Huffington Post).

Todas las entrevistas fueron telefónicas, duraron entre 20 y 40 minutos, y fueron grabadas y transcritas. Tuvieron lugar entre los meses de octubre y diciembre de 2018 en Barcelona y Nueva York y se utilizó una misma guía de entrevista (ver tabla 2). Además, se han incluido citas de una entrevista publicada en The New York Times con motivo del anuncio del nombramiento de Jessica Bennet como editora de género. Este se considera que es el primer caso en que un medio tradicional generalista de referencia crea esta figura y dada su trascendencia hemos estimado oportuno usar citas extraídas de esa entrevista (se contactó directamente a la editora pero rechazó ser entrevistada).

TABLA 1

Guía de entrevista

La figura de la editora de género

1. ¿Cuáles son los objetivos que persigue la empresa con la creación de esta figura? ¿Rol permanente o puntual?

2. ¿Cuáles son las causas para que aparezca ahora esta figura? ¿Cree que hay una relación entre la creación de esta figura-enfoque y el movimiento \#metoo? ¿Cambio de paradigma o tendencia de marketing?

3. ¿Cuáles son las consecuencias desde que su medio ha incorporado esta figura? ¿Cómo se ha plasmado en los contenidos?

4. ¿Cuáles son las principales alertas dentro de su medio?

Desarrollo de audiencias y comunidad

1. ¿Han detectado una comunidad de lectores interesados específicamente en esta cuestión?

2. ¿Qué datos puede compartir acerca de esta comunidad (demografia)? ¿Consumen otros contenidos del medio o se focalizan en "piezas feministas»?

3. ¿Estaría de acuerdo con la afirmación de que la creación de esta figura responde también a una estrategia empresarial, que persigue conectar con una comunidad de lectores determinada?

\section{Igualdad en el medio de comunicación estudiado}

1. ¿Siente el apoyo de su empresa y de la redacción? ¿Ha habido reticencias en el momento de creación e implantación de esta figura?

2. ¿Hay mujeres en el nivel más alto de toma de decisiones de su medio? ¿Y en los cargos intermedios? (Jefes de sección, redactores jefe...)

3. ¿Hay igualdad de género en las condiciones laborales? Principalmente: igualdad de salarios y estabilidad.

4. ¿Hay una correcta representación de la mujer?

Fuente: elaboración propia. 
Este estudio tiene varias limitaciones importantes. En primer lugar, no se trata de una muestra probabilística o representativa. Somos conscientes de que cinco entrevistas aportan una perspectiva muy pequeña, modesta e incluso sesgada, pero en términos cualitativos la muestra permite un enfoque en profundidad de las experiencias de las distintas profesionales entrevistadas. Constatamos la necesidad de una investigación cualitativa y cuantitativa más amplia, pero este trabajo proporciona, según nuestra opinión, información valiosa acerca de percepciones, oportunidades y retos que actualmente las redacciones de los medios generalistas están viviendo en España y en Estados Unidos en relación a ciertos roles que incorporan el prisma de género. En segundo lugar, la muestra es muy dispar en lo que se refiere al tipo de medio. Se incluyen medios tradicionales, medios nativos digitales, medios públicos, corporaciones privadas... somos conscientes de esta disparidad pero en lugar de considerarla un impedimento la percibimos como una oportunidad para originar un debate necesario para las ciencias de la comunicación y la información.

Finalmente queremos expresar que este es un estudio preliminar.

\section{Resultados}

La relación entre feminismo y medios de comunicación no es algo nuevo. No importa si hablamos de prensa en papel o de plataformas como Twitter o Facebook. Los movimientos sociales tradicionalmente han visto en los medios un aliado o una herramienta propagandística, dependiendo de con quién se hable. Esta investigación se centra en una realidad nueva muy específica: el rol de las editoras de género, así como de otros perfiles profesionales satélite que refuerzan la idea de que las noticias también se pueden explicar con lentes violeta. Hemos visto anteriormente que los medios de comunicación generalistas tradicionalmente se asimilan a la esfera pública, usualmente reservada al sexo masculino, y una de las principales consecuencias es que muchos medios cuentan con una audiencia mayoritariamente masculina. ¿Qué pasaría si esas mismas noticias se narraran desde una perspectiva de género más incluyente? ¿Qué consecuencias tendría a nivel de contenido? ¿Promoverían la igualdad de condiciones laborales en aquellas redacciones con figuras como la de la editora de género? ¿Y por qué ahora se suceden estos cambios?

El análisis de las entrevistas ha permitido identificar dos áreas de interés: (1) descripción de la figura de la editora-redactora de género (aproximación, objetivos, apoyo institucional), (2) cuestionamiento del cambio de paradigma o estrategia comercial. 


\subsection{DesCripción DE LA Figura DE LA EDITORA DE GÉNERO}

\subsubsection{Aproximación}

Todas las entrevistas mantenidas coinciden en señalar que la figura de la editora de género (inclusive las posiciones satélite) es un rol que debe buscar la transversalidad en la cobertura de noticias con una perspectiva de género. Este es un cambio fundamental respecto al modus operandi de la mayoría de medios generalistas en los que los «temas de género» suelen tener trato diferenciado en una sección específica pensada principalmente para un público femenino. Sin embargo, en palabras de la propia Jessica Bennet (The New York Times), su posición se crea para asegurar que los contenidos de género se encuentren en todo el periódico, algo que en sí mismo aparenta ser un reto incluso para ella misma "nuestro contenido de género existirá en cada sección del periódico y se producirá en todos los medios. Lo que realmente hace que mi trabajo sea relativamente desalentador, porque significa que soy un poco como un editor itinerante que trabaja en todas las secciones y departamentos... pero también creo que ese enfoque es crucial. Porque estamos tratando de expandir y mejorar orgánicamente la cobertura de estos temas en cada sección, de manera que pase inadvertido, porque el género no debería tener una sección propia. Sigo diciendo: sabré que tengo éxito en este rol cuando mi posición ya no sea necesaria».

En este mismo sentido se pronunciaba Ana Requena (eldiario.es) cuando afirmaba que «trabajar por incorporar la perspectiva de género en el medio es el principal objetivo que perseguimos con la creación de esta figura de redactora jefa de género. No se trata de hacer un cajón de sastre donde almacenar los contenidos "por» $y$ "para» mujeres, sino que buscamos una mirada transversal que atraviese todo el medio. Para ello, lo primero es concienciar a todos los redactores en no cometer errores básicos en el tratamiento de las violencias machistas (las mujeres no mueren, las matan; no se debe culpabilizar a las víctimas y exculpar a los agresores, etc.). Así como ofrecer temas interesantes que pueden $y$ deben ser abordados con perspectiva de género y van más allá de los tradicionales temas de siempre "por» y "para» mujeres».

Alicia Gómez (RTVE) también expresaba la apuesta de su medio por la transversalidad «los contenidos de igualdad, la mirada de igualdad, la conciencia de igualdad no se pueden circunscribir a fechas concretas que vienen marcadas por la agenda sino que es algo transversal que tiene que venir de una concienciación de todos los empleados... queremos atravesar los contenidos informativos para imponer, entre comillas, la igualdad a través de los hechos». Pilar Álvarez (El País) lo resume así: «mi labor será transversal a todas las secciones. No se trata solo de escribir más historias sobre mujeres, sino de incluir más mujeres en las historias». 


\subsubsection{Objetivos}

¿Cuáles son los objetivos estos roles? Este también ha sido un aspecto crucial en las entrevistas, comprender de primera mano qué es lo que este tipo de posiciones persiguen en última instancia. Las distintas entrevistas permiten afirmar que fundamentalmente este tipo de roles se sustentan sobre el trinomio responsabilidad-cobertura-reivindicación.

Responsabilidad porque la incorporación de figuras como la editora de género, la editora de igualdad o la columnista de género es una obligación de la profesión periodística. En este sentido todas las entrevistadas se muestran unánimes, para Monica Hesse (The Washington Post), dar cobertura a temas de género es en realidad hablar de un cierto modo de entender la sociedad en que vivimos «cuando hablamos de género, realmente estamos hablando de relaciones humanas y de la sociedad, cómo llegamos al momento actual, qué es injusto al respecto, qué es lo que no funciona... así que cuando hablamos de género realmente hablamos del mundo y de por qué está configurado de cierta forma. Esa es una misión central para el periodismo, o debería serlo... para entender por qué somos como somos». Alanna Vagianos (The Huffington Post) admitía que para ella el género es algo inherente en el propio modo de cubrir las noticias «el género está en cada artículo de noticias que escribimos. El género es fundamental para entender la política, para entender la cultura pop, el activismo y la justicia social ... necesitamos más personas que tengan esta experiencia en la redacción para resaltar estos temas tan importantes».

Para Alicia Gómez (RTVE) en su caso es una obligación en tanto que trabaja para un medio público: "Somos mujeres, somos la mitad del mundo y debemos estar bien representadas en las coberturas mediáticas. Esto siempre lo hemos intentado en los informativos, pero ahora lo hacemos todavía más, con una especial incidencia en economía y deportes que son secciones muy masculinizadas. También ha aumentado mucho el número de noticias acerca de las mujeres y la igualdad, para concienciar. La televisión pública tiene la responsabilidad de cuidar estos aspectos, y como servicio público debe tener este rol».

La cobertura informativa de temas tradicionalmente silenciados como son las agresiones sexuales o la discriminación por razones de género son otra de las funciones básicas de este nuevo rol profesional. Las entrevistadas consideran la creación de este tipo de roles algo necesario e inherente a su propia profesión, pero ¿tiene impacto en la cobertura mediática? Según las entrevistas realizadas, sí, y el principal impacto es el hecho que se da visibilidad a noticias que muchas veces pasaban desapercibidas por considerarse tabú, eran silenciadas por ser políticamente incorrectas o no se consideraban de interés para la audiencia mayoritaria del medio en cuestión. Según Monica Hesse (The Washington Post) estas figuras no solo llevan a la mesa temas nuevos sino que tienen impacto en el modo en que se cubren los mismos eventos «roles como el mío han tenido impacto en el contenido, soy una columnista y no una reportera por ejemplo, por lo que puedo cubrir las noticias de diferentes maneras. Si cubrimos el caso Brett 
Kavanaugh aquí en los EE. UU., en lugar de simplemente escribir artículos explicando lo que sucedió hoy etcétera, puedo tomar una lente más amplia y pensar más en el significado... ¿qué dice eso acerca de nuestros sesgos? ¿qué significa para nuestro país? ¿cómo pensamos sobre el asalto a mujeres? Creo que estos roles abren nuevas posibilidades a nivel de temas tratados $y$ cómo tratarlos».

En la misma línea se pronunciaba Bennett (The New York Times) cuando le preguntaron acerca del tipo de cobertura y de la posibilidad de dar voz a cuestiones (y a actores) tradicionalmente silenciados «estamos pensando en estos temas realmente de manera holística, se trata del tipo de cobertura $y$, en ocasiones, de elevar algunas de las voces sub-representadas. También se trata de analizar críticamente nuestro propio modo de cubrir la información y de pensar en el tono, los elementos sutiles del lenguaje y las imágenes que acompañan las historias y las fuentes.»

Pilar Álvarez (El País) se refería así: "para entender el cambio que ha habido en los últimos años solo hay que mirar cómo los medios cubrimos el caso Strauss-Kahn el año 2015 y cómo lo hemos hecho ahora con Weinstein. Strauss-Kahn y Weinstein son dos casos similares en el sentido de que un hombre poderoso es acusado de abusos sexuales. En su entorno, todo el mundo sabía lo que hacían y todo el mundo hablaba. El caso Strauss-Kahn, por parte de los medios, se abordó sólo desde la vertiente política. Ahora esto sería impensable».

El carácter reivindicativo es el tercer elemento que, según las profesionales entrevistadas, define a los distintos roles de género descritos en este artículo. Más allá de los factores de responsabilidad y de cobertura informativa (visibilidad) las editoras de género, así como las reporteras y columnistas de género o igualdad, también tienen un elemento reivindicativo. Éste es importantísimo porque estas figuras tienen un papel disruptivo a nivel externo (audiencias-sociedad) pero también interno en el seno de la propia industria de los medios. Así, se persiguen condiciones laborales igualitarias, siendo el objetivo final la transformación del medio mediante factores como la igualdad de género en la redacción (principalmente masculina), mitigar la discriminación salarial, y romper con el techo de cristal (la mayoría de entrevistadas afirma que los cargos de toma de decisión están en manos de hombres). En palabras de Alicia Gómez (RTVE) «... cuando subes a las plantas nobles solo te encuentras corbatas en los despachos y eso no es lógico...».

Monica Hesse (The Washington Post) iba más allá en sus reflexiones al considerar que roles como el suyo estaban relacionados con el modelo de mundo que los medios reivindican "para mi, esto va más allá de escribir sobre el movimiento \#metoo, el número de mujeres en el congreso o la brecha salarial... realmente es un tema clave del mundo en el que vivimos y por qué se percibe de cierta forma.»

Hablando de reivindicaciones, una reflexión candente es la necesidad de incorporar voces que superen las barreras binarias de género, un tema de debate en Es- 
tados Unidos donde por ejemplo el estado de Nueva York ha aprobado una ley en favor de los certificados de nacimiento no binarios; a partir de enero de 2019 los padres y madres podrán elegir si identifican a su bebé como sexo masculino, femenino o neutro ${ }^{2}$. Así, Alanna Vagianos (The Huffington Post), insistía en que «tener voces más diversas dentro del ámbito del género es definitivamente importante. Cuando hablamos de mujeres, las personas suelen pensar en mujeres, pero uno de los temas relacionados con el equilibrio de género tiene que ver con la misoginia. Cuando hablamos de género, obviamente hay más que solo dos géneros. Muchos de los problemas están relacionados con los hombres, por lo que si podemos lograr que más hombres y personas no binarias hablen de género, eso sería maravilloso.» En la misma línea se pronunciaba Jessica Bennet (The New York Times) al afirmar que "la realidad es que los medios de comunicación han sido creados durante mucho tiempo por $y$ para los hombres blancos. Es un hecho. No creo que sea parcial o, de alguna manera, no neutral, decir que ya no es el mundo en el que vivimos, y que nuestra cobertura debería reflejar eso".

Las editoras de género españolas también quieren incorporar voces para visibilizar la realidad de otras mujeres más allá de las de raza blanca de mediana edad y con estudios superiores. Para ello, existe un debate interno para afinar bien su rol. Alicia Gómez (RTVE) señalaba que "al final, ¿de qué estamos hablando? ¿de cubrir la realidad de las mujeres de la Índia o solo de las que viven en un contexto acomodado? ¿de los derechos de las mujeres o incluimos aquí también los del colectivo LGTBI? Me preocupa generar demasiadas expectativas y no cumplirlas». Ana Requena (eldiario.es) apunta: "como editoras de género de medios occidentales, tenemos nuestros sesgos, pero trabajamos para incorporar cuantas más voces mejor sin perder de vista que nos dirigimos a un público mayoritario. Sé que necesitamos más mujeres columnistas racializadas, por ejemplo».

\subsubsection{Apoyo institucional}

Todas las entrevistadas se sienten apoyadas por su equipo, la redacción y la dirección del medio. La posición de Alicia Gómez (RTVE) tuvo el apoyo de la redacción y recibe el mismo trato que otras posiciones similares, participando de las reuniones de directivos y editores de informativos: "participo de las reuniones de previsiones dónde están los editores y las jefaturas de área de manera que no solamente escucho temas que ellos proponen sino que también intervengo si hay que tirar de las orejas a alguien." Así mismo se pronunciaba Pilar Álvarez (El País) cuando se le preguntó sobre las reacciones de sus compañeros cuando se anunció la creación de su posición «... la creación de la figura, de la que ya existen ejemplos en algunos medios anglosajones, nace de una

2 <https://www.cnn.com/2019/01/03/health/new-york-city-gender-neutral-birthcertificate-trnd/index.html?utm_source $=$ CNN+Five+Things\&utm_campaign $=8017801 \mathrm{fa} 2-E M A I L \_$ CAMPAIGN_2019_01_04_12_41\&utm_medium=email\&utm_term=0_6da287d761-8017801fa298149997> [Consulta: 3 de abril de 2019] 
propuesta de las periodistas de El País recogida y escuchada por el director, Antonio Caño", reacciones similares a las descritas por Ana Requena (eldiario.es) «... hay un empuje dentro de las redacciones, antes quizás eran periodistas solas intentando generar cambio dentro las redacciones, pero ahora las más jóvenes suben con más fuerza y formación, y el resto de la redacción lo recoge bien. En mi caso, he tenido el apoyo tanto de la dirección como de la redacción...» Las periodistas estadounidenses consultadas también coinciden en apuntar que se sintieron apoyadas desde el primer día. Para Monica Hesse (The Washington Post) fue crucial no tener que presionar a nadie para que su posición fuera creada «este es un cargo que la dirección del periódico creó y yo simplemente apliqué, no tuve que presionar a nadie... estos son unos temas que se querían cubrir» mientras que para Alanna Vagianos (The Huffington Post) seguramente la creación de esta figura va asociada al hecho que trabaja para un medio que se autodefine como liberal y progresista «en general, han sido muy comprensivos, este es un medio muy progresista. Recuerdo el caso de Brock Turner, el nadador de Stanford, que se destapó en 2015, antes del movimiento \#metoo... algunos de mis colegas me dijeron que la audiencia no estaba lista para esa historia y de alguna manera esto me hizo retroceder un poco... pero creo que esto definitivamente ha cambiado con \#metoo. Creo que, en general, como reportera hay un espacio abierto para cubrir estas historias y me sentí apoyada desde el primer día.»

\section{2. ¿CAMbio DE PARAdigma O ESTRATEgia COMERCiAL?}

Identificar si la proliferación de estos nuevos roles profesionales con perspectiva de género es para los medios un cambio de paradigma o una estrategia comercial ha sido un elemento crítico en este estudio de caso. Hemos buscado distintas perspectivas, combinando preguntas en el cuestionario que permitieran discernir si se trataba de un planteamiento o del otro. Finalmente, concluimos que las distintas entrevistadas no consideran que sea una iniciativa excluyente, dicotómica. Al contrario, la percepción unánime es de que se trata de una oportunidad profesional con beneficios empresariales y para los lectores. Monica Hesse (The Washington Post) sugería que cargos como el suyo representan ante todo una oportunidad para el lector «según mi modo de entender, definitivamente no es una estrategia de marketing ... para mí, y creo que para mis editores, fue una oportunidad para tener conversaciones guiadas sobre ciertos temas, para tratar de ofrecer herramientas de interpretación de temas que durante mucho tiempo habian sido confusos para mucha gente.» Alanna Vagianos (The Huffington Post) se mostró incluso un tanto sorprendida por la pregunta puesto que el medio para el que ella trabaja está considerado muy progresista desde sus inicios «siempre hemos tenido estos roles en el Huffington Post. Siempre ha sido un pilar de la forma en que este periódico $\mathrm{cu}$ bre las noticias, desde su fundación. Pero es cierto que más y más personas están cubriendo estos temas ahora.»

En el caso de los medios españoles, Ana Requena (eldiario.es) señaló que empresarialmente el rol de redactora jefa de género otorga un prestigio añadido al me- 
dio al tiempo que permite alcanzar un nicho de audiencia que es significativamente menor: "... en los últimos años hay un auge feminista general en la sociedad, que ha empujado también a que sucedan cosas dentro de los propios medios de comunicación. A eso hay que sumar el auge de las redes sociales y la posibilidad de las audiencias de contactar directamente con los medios... Esto hace a los medios más conscientes de ciertos errores, entre ellos la necesidad de tratar mejor los temas que interesan a las mujeres (...) Los medios de comunicación, especialmente los progresistas, no son ajenos a este momento $y$ han visto que cultivar una comunidad de mujeres interesa también, aunque no únicamente, a nivel empresarial». Según Alicia Gómez (RTVE) «... hay un cierto sentido de oportunidad porque hay un estado de gracia en lo que es esta tercera revolución feminista... creo que sí está en la cabeza de alguien que esto es únicamente una estrategia y que luego se puede pinchar el globo, honestamente pienso que se equivocan. Cuando los avances y las mejoras reparan situaciones que se vienen arrastrando desde décadas y que son injustas, luego ya se quedan...».

Las distintas entrevistadas consideran estos cambios estructurales una oportunidad con beneficios empresariales, pero, ¿cómo afectó el movimiento \#metoo? ¿fue un pistoletazo de salida? Encontramos disparidad de opiniones, las entrevistadas de medios españoles coinciden en vislumbrar cierta correlación entre este movimiento y la irrupción de la figura de la editora de género, pero las entrevistadas estadounidenses no lo ven así. Alicia Gómez (RTVE) insistía en el hecho de que "hace un año esto era impensable, esto es el 'reflujo' de todo lo que empezó en Estados Unidos con el \#metoo aunque había un caldo de cultivo importante, una sociedad preparada para el cambio, para la feminización de los contenidos y para el empoderamiento de la mujer... pero la sociedad estaba preparada dormida, necesitábamos algo que nos despertara de una especie de resignación social... el movimiento \#metoo fue un interruptor.» En la misma línea se pronunciaba Pilar Álvarez (El País) al afirmar que es innegable el contexto social con la creación de la corresponsalía de género: "la masiva participación en las movilizaciones del 8 de marzo en España, las protestas por la sentencia de La Manada o las denuncias contra el acoso sexual del \#MeToo y el \#Cuéntalo aquí en España dan cuenta del momento que atraviesa el feminismo en este momento».

Sin embargo, según Alanna Vagianos (The Huffington Post) temas como el acoso sexual ya eran parte de la dieta mediática antes de la irrupción de esta campaña «No creo que haya una correlación porque el \#metoo fue un gran movimiento, pero muchos medios ya estaban hablando sobre las agresiones sexuales. No creo que eso haya llevado a la creación de más empleos, pero sí creo que hay reporteros de noticias generales que no estaban cubriendo temas de género y ahora lo están haciendo mucho más." Monica Hesse (The Washington Post) admitía que su posición se creó al mismo tiempo que surgía el movimiento \#metoo aunque no considera que fuera una causa-efecto "No puedo hablar sobre el por qué se creó mi posición, aunque el trabajo se publicó alrededor del momento en que el movimiento \#metoo se convertía en parte del debate nacional. Pero en términos de por qué quería tener el trabajo y por qué mis editores y yo lo considerábamos tan importante, lo relevante es que finalmente éramos un país que mantenía una conversa- 
ción franca sobre género y de una manera que realmente no habíamos visto antes, no había precedentes... por lo que empezamos a buscar oportunidades para tener este tipo de conversaciones en un formato diferente."

\section{Conclusiones y futuras líneas de investigación}

El análisis de las entrevistas permite dibujar una aproximación preliminar a los principales retos y oportunidades de la figura de la editora de género. Como ya se ha apuntado en la nota metodológica, la muestra analizada es muy pequeña y esto presenta serias limitaciones para el estudio, sin embargo, este micro análisis permite plantear algunas ideas para el debate y a la reflexión, recogidas a continuación según las categorías utilizadas en la guía de entrevista:

\section{LA FIGURA DE LA EDITORA DE GÉNERO}

Motivaciones de los cambios estructurales. La muestra analizada no permite corroborar o rechazar la hipótesis principal de este estudio que sugería una posible motivación oportunista de los medios estudiados en la creación de la figura de la editora de género. Las distintas profesionales entrevistadas coinciden en señalar que el caso Harvey Weinstein (2016), el movimiento \#metoo y la huelga feminista convocada en España el 8 de marzo de 2018 han sido catalizadores de este cambio estructural pero no hay evidencias que sugieran que la creación de este rol responda a una iniciativa comercial o de marketing.

Apoyo institucional. Se ha detectado la percepción de que las entrevistadas consideran que hay un apoyo incondicional por parte de sus compañeros de redacción, los editores jefe y los distintos órganos directivos.

Responsabilidad profesional. La creación de la figura de la editora de género es un gesto disruptivo pero también representa una oportunidad para repensar una profesión (la periodística) que juega un papel fundamental en la sociedad. El apoyo de las nuevas generaciones de periodistas, educadas en la era de la información y la comunicación, ha sido crítico.

\section{DesarRollo DE AUDIENCIAS Y COMUNIDAD}

Audiencia. Se corrobora el apetito de la audiencia de los distintos medios analizados de cobertura de noticias con lentes violeta. A nivel estratégico, las editoras apuestan por una aproximación holística y transversal. 
Riesgo de marginalización de contenidos. Se confirma la hipótesis inicial según la cual a menudo la cobertura de temas relacionados con el género se encasilla en páginas web contenedores, algo que contradice el primer punto según el cual las editoras de género deben velar por la transversalidad de su rol$^{3}$; algo distinto es el uso de etiquetas o hashtags para la promoción e indexación de contenidos.

Segmentación. Los lectores de las piezas y/o secciones editadas por las editoras de género tienen una audiencia mayoritariamente femenina, confirmándose así otra de las hipótesis apuntada al inicio del artículo. Así mismo, los reporteros y los editores son principalmente mujeres, constatándose la necesidad de trabajar por un entorno más inclusivo en el que la cobertura mediática con la óptica de género no sea percibida como una temática hecha por y para mujeres.

\section{IGUALDAD EN EL MEDIO ESTUDIADO}

Apoyo de la empresa y de la redacción. Todas las entrevistadas señalan que han recibido el apoyo incondicional de compañeros así como de supervisores y jefes directos. Entienden la creación de este rol como parte de una decisión estratégica del medio.

Condiciones laborales y representación de género. Pese a preguntar directamente acerca de las condiciones laborales y de la representación de la mujer en la redacción, sentimos que este debate se ha diluido en opiniones muy personales y ninguna de las entrevistadas ha proporcionado datos que sustenten una u otra percepción. En el caso de las entrevistadas americanas adoptaron una posición extremadamente corporativa y nos refirieron a sus supervisores alegando que no se sentían cómodas discutiendo este tipo de detalles.

Este estudio preliminar ha abierto ciertos interrogantes acerca de las motivaciones y las implicaciones que la figura de la editora de género tiene. Futuras investigaciones deberán contemplar el estudio de otros medios, tanto españoles como internacionales. Una de las primeras acciones que vamos a llevar a cabo es la ampliación de la muestra para recoger más opiniones y de medios más diversos, tanto geográficamente como ideológicamente. También es necesario establecer una tipología de medios según soporte puesto que anticipamos resultados distintos para los medios

\footnotetext{
3 Algunos ejemplos son: RTVE - Portal Todxs por igual <http://www.rtve.es/noticias/ todxsporigual/> [Consulta: 3 de abril de 2019]. EL PAÍS - Cuenta de Twitter@elpais_fem <https:// twitter.com/elpais_fem> [Consulta: 3 de abril de 2019]
} 
nativos digitales con respecto de los tradicionales. Una tercera sugerencia para futuras investigaciones es incorporar las percepciones de la audiencia mediante focus groups y encuestas.

\section{Referencias bibliográficas}

Anderson, C. W., Bell, E. J. y Shirky, C. (2012). Postindustrial Journalism. The Tow Center for Digital Journalism. Columbia University. Recuperado de https:// academiccommons.columbia.edu/doi/10.7916/D8N01JS7

Bakker, P. (2014). Mr. Gates returns: Curation, community management and other new roles for journalists. Journalism studies, 15(5), 596-606.

Bell, E.J., Owen, T., Brown, P.D., Hauka, C. y Rashidian, N. (2017). The Platform Press: How Silicon Valley Reengineered Journalism. The Tow Center for Digital Journalism. Columbia University. Recuperado de https://academiccommons.columbia.edu/ doi/10.7916/D8R216ZZ

Benesch, C. (2012). An Empirical Analysis of the Gender Gap in News Consumption. Journal of Media Economics, 25(3), 147-67. doi:10.1080/08997764.2012.700976.

Boczkowski, P. (2004). Digitalizing the News: Innovation in Online Newspapers. Estados Unidos: The MIT Press.

Caminero-Fernández, L. y Sánchez-García, P. (2018). El perfil y formación del ciberperiodista en redacciones nativas digitales. Hipertext.net: Revista Académica sobre Documentación Digital y Comunicación Interactiva, (16), 4-15.

Castells, M. (2009) Comunicación y poder. Madrid: Alianza Editorial.

Cobos, T.L., (2011). Y surge el community manager. Razón y palabra, 16(75). Recuperado de http://www.razonypalabra.org.mx/N/N75/varia_75/varia2parte/15_Cobos_V75. pdf

Deuze, M. (2011) Managing Media Work. Thousand Oaks, California: SAGE Publications.

Domingo, D. (2011). Managing Audience Participation: Practices, Workflows and Strategies. Participatory Journalism: Guarding Open Gates in Online Newspapers, ed. Jane B. Singer et al. New York: Wiley-Blackwell.

Franks, S. (2013). Women and Journalism. London: I.B.Tauris \& Co. Ltd.

Gallego, J. (2013). De reinas a ciudadanas: medios de comunicación, ¿motor o rémora para la igualdad? Madrid: Aresta.

Gallego J. (2003). Producción informativa y transmisión de estereotipos de género en la prensa diaria. Comunicación y sociedad, XVI (2), 49-66.

García Saiz, L. (2018) Análisis de la situación laboral de las periodistas españolas. Cuestiones de género: de la igualdad y la diferencia, 13, 7-27.

Gillmor, D. (2004) We the media: grassroots journalism by the people, for the people. Beijing: O'Reilly. 
Guallar, J. y Codina, L. (2018). Journalistic content curation and news librarianship: Differential characteristics and necessary convergence. El profesional de la información, 27(4), 778-791. Recuperado de https://doi.org/10.3145/epi.2018.jul.07

Hazard, L. (2018). Here's what the Financial Times is doing to get bossy man voice out of (okay, less prominent in) its opinion section. Nieman Lab. Recuperado de http://www.niemanlab. org/2018/09/heres-what-the-financial-times-is-doing-to-get-bossy-man-voice-outof-okay-less-prominent-in-its-opinion-section/

Holton, A. E., Lewis, S. C. y Coddington, M. (2016) Interacting with Audiences. Journalism Studies, 17(7), 849-859.

Larsson, A. O. y Andrea I. K. (2017). Beyond 'J-Tweeters'. Journalism Practice, 11(6), 689704. doi: $10.1080 / 17512786.2016 .1181983$

McNamara, B. (2017). Meet The New York Times's first gender editor. Teen Vogue. Recuperado de https://www.teenvogue.com/story/new-york-times-first-gender-editor

McCracken, K., FitzSimmons, A., Priest, S., Girstmair, S., y Murphy, B. (2018). Gender Equality in the Media Sector. Directorate-General for Internal Policies. European Parliament. Recuperado de http://www.europarl.europa.eu/RegData/etudes/ STUD/2018/596839/IPOL_STU(2018)596839_EN.pdf

Pavlik, J. V. (2013) Innovation and the future of journalism. Digital Journalism, 1(2), 181193. doi: $10.1080 / 21670811.2012 .756666$

Pérez-Soler, S. y Micó-Sanz, J. L. (2015). El mito de la conversación global. Usos de Twitter en las redacciones periodísticas catalanas y belgas. El profesional de la información, 24(3), 246-255.

Piet, B. (2012). «Aggregation, Content Farms and Huffinization: The Rise of Low-pay and No-pay Journalism.» Journalism Practice 6 (5-6), 627-637.

Prieto Sánchez, Cristina. (2018). ¿Quiénes hablan en las noticias? Desequilibrio de género en las fuentes informativas de la prensa de proximidad. Zer: Revista de Estudios de Comunicación, 23(45), 161-184.

Rashidian, N., Brown, P.D. y Hansen, E. (2018). Friend and Foe: The Platform Press at the Heart of Journalism. The Tow Center for Digital Journalism. Columbia University. Recuperado de https://www.cjr.org/tow_center_reports/the-platform-press-at-theheart-of-journalism.php

Roca, M. (2014). Shifting to Digital: Difficulties, Challenges, and Opportunities-A Qualitative Interview Study of Practitioners' Experiences in the US. Advertising \& Society Review, 15(3).

Rosentiel, T. (2008). Where Men and Women Differ in Following the News. Pew Research Center. Recuperado de http://www.pewresearch.org/2008/02/06/where-men-andwomen-differ-in-following-the-news

Spayd, L. (2017). The Declining Fortunes of Women at The Times. The New York Times. Recuperado de http://www.nytimes.com/2017/03/04/public-editor/the-decliningfortunes-of-women-at-the-times.html 
The New York Times. (2017). Jessica Bennett, our new gender editor, answers your questions. Recuperado de https://www.nytimes.com/2017/12/13/reader-center/jessica-bennettour-new-gender-editor-answers-your-questions.html

Toff, B. J., y Palmer, R. A. (2018). Explaining the Gender Gap in News Avoidance: «NewsIs-for-Men» Perceptions and the Burdens of Caretaking. Journalism Studies. Recuperado de https://doi.org/10.1080/1461670X.2018.1528882

Zeisler, A. (2018). Media's Gender Gap. Investigating relationships between women's news production and consumption. USC Annenberg Norman Lear Center. Recuperado de http:// mediaimpactproject.org/uploads/5/1/2/7/5127770/womennewsroom.pdf

Toff, B. J., y Palmer, R. A. (2018). Explaining the Gender Gap in News Avoidance: «NewsIs-for-Men» Perceptions and the Burdens of Caretaking. Journalism Studies. Recuperado de https://doi.org/10.1080/1461670X.2018.1528882

Zeisler, A. (2018). Media's Gender Gap. Investigating relationships between women's news production and consumption. USC Annenberg Norman Lear Center. Recuperado de http:// mediaimpactproject.org/uploads/5/1/2/7/5127770/womennewsroom.pdf 
